\title{
Age-Related Changes in Heart Rate during Copulatory Behavior of Male Rats
}

\author{
Misao TERADA ${ }^{1)}$, Seiko WATO ${ }^{2)}$, Pacharaporn KROMKHUN ${ }^{2,6)}$, Haruo HASHIMOTO ${ }^{3)}$, \\ Kazumi TANIGUCHI ${ }^{4)}$, Fujiro SAKURAI ${ }^{5)}$, Makoto YOKOSUKA ${ }^{2)}$, \\ Gyorgy G. NAGY ${ }^{7}$, and Toru R. SAITO ${ }^{2)}$

\begin{abstract}
${ }^{1)}$ Division of Laboratory Animal Science, Nippon Medical School, Bunkyo-ku, Tokyo 113-8602, ${ }^{2)}$ Department of Comparative and Behavioral Medicine, Nippon Veterinary and Life Science University, Musashino-shi, Tokyo 180-8602, 3) Animal Resource Center, Central Institute for Experimental Animals, Kawasaki-shi, Kanagawa 216-0001, ${ }^{4)}$ Department of Veterinary Anatomy, Kitasato University, Towada-shi, Aomori 034-8628, ${ }^{5}$ Department of Animal Science, Teikyo University of Science and Technology, Uenohara-shi, Yamanashi 409-0193, Japan, ${ }^{6}$ Department of Physiology, Kasetsart University, Bangkok 10900, Thailand, and ${ }^{7)}$ Department of Human Morphology and Developmental Biology, Semmelweis University, Budapest H-1094, Hungary
\end{abstract}

\begin{abstract}
As members of Western societies age, sexual function of older (elderly) individuals becomes an important issue, particularly for men. Specifically, copulatory behavior increases circulatory load, which may be related to reports of cardiac sudden death following ejaculation. To further examine this relationship, we compared heart rate (HR) before and after ejaculation in 48-week-old (aged) and 10-week-old (young) male rats. As compared with resting HR, $\mathrm{HR}$ after ejaculation was increased by $54.2 \pm 3.5$ and $41.7 \pm 2.7 \%$, respectively, among aged and young male rats. These values were significantly higher than baseline levels $(P<0.01)$. The increases in $\mathrm{HR}$ at each time point during copulation were significantly higher in aged male rats than in young male rats $(P<0.05$ or $P<0.01)$. We also studied decreases in HR following ejaculation and found that aged male rats had a significantly higher HR at 1 and 2 min after ejaculation than young rats $(P<0.01)$. These results suggest that the circulatory load on the aged rat heart is greater than that on a young rat heart during copulatory behavior, especially at ejaculation. In addition, the decrease in HR in aged rats after ejaculation was more gradual than in young male rats. These results suggest that there is a higher risk of sudden cardiac death during sexual behavior in older males.
\end{abstract}

Key words: ageing rat, copulation, heart rate

As Western societies age, sexual function of older (elderly) individuals becomes an important issue, particularly for men. Sexual function in male mammals is roughly classified into fertility and sexual potency. Sexual potency has been examined in detail within West- ern countries [15]; however, little research on this subject has been conducted in Japan. There is much interest worldwide in developing an animal model of human potency $[16,17]$.

Libido and copulatory behavior decline in humans and

(Received 27 October 2008 / Accepted 18 March 2009)

Address corresponding: T. R. Saito, Department of Comparative and Behavioral Medicine, Nippon Veterinary and Life Science University, 1-7-1 Kyonan-cho, Musashino-shi, Tokyo 180-8602, Japan 
animals with increasing age $[5,8,10,11,18,22]$. Saito et al. [25, 27] demonstrated that penile reflexes and copulatory behavior in 44-week-old male rats are decreased as compared with 10 -week-old male rats. Cardiovascular sudden death of elderly men during sexual activity has been reported $[19,23,29]$ and is considered to be a serious problem. In previous studies, we reported changes in heart rate (HR) of young adult male rats and horses during copulation, as measured with a telemetry system. These results demonstrate the involvement of the autonomic nervous system $[26,28]$ and suggest that copulatory behavior increases the load on the circulatory system. However, very few attempts have been made to measure HR during sexual behavior in elderly people. Furthermore, little research on this topic has been conducted in Japan. The aim of the present study was to investigate how ageing influences HR during copulatory behavior in males using a rat model.

Experimental animals: On the basis of previous studies and observations [25, 27], we used in our experiments specific-pathogen-free male Wistar-Imamichi rats that were 48 weeks old (aged, $n=4$ ) and 10 weeks old (young, $\mathrm{n}=6$ ), and 10-week-old female rats (raised in our laboratory). All animals were acclimatized in an animal room (12-h light/dark cycle and a temperature of $23-25^{\circ} \mathrm{C}$ ) and were given free access to water and food. All procedures were in accordance with the NIH Guide for the Animal Care and Use Committee of Nippon Veterinary and Life Science University.

Telemetry implant and measurements: One week before the experiments, each male rat was anesthetized with isoflurane and secured in a prone position on the operating table. A 5-cm incision was then made in the dorsal skin. A telemetric transmitter (TA11CTA-F40, Data Sciences, St Paul, MN, USA) for electrocardiography (ECG) was immersed in sterile saline at room temperature for $10 \mathrm{~min}$ and then implanted subcutaneously in the neck, as described by Ishii et al. [14]. Paired wire electrodes were placed under the skin of the thorax (chest bipolar ECG lead). The AM signals transmitted from the telemetric transmitter were amplified as analogue ECG waveforms by a receiver (RA1310, Data Sciences) and then analyzed (Dataquest III, Data Sciences). HR was measured every minute by the data editing function of the ECG analyzer.
Observation of copulatory behavior: Each male rat was placed in an observation cage $(430 \times 3,300 \times 265$ $\mathrm{mm}$ ) under a red light and 5 min later a female rat that was sexually receptive (as determined by the presence of pro-estrous features on vaginal smears taken after two cycles of the 4-day estrous cycle) was introduced into the same cage. Copulatory behavior-induced changes in HR were measured for $1 \mathrm{~h}$ and measurements began $1 \mathrm{~h}$ after the male rat entered the cage.

Statistical analysis: All data were recorded up to the second ejaculation of each rat and are presented as means \pm SE. Statistical analyses of the data were performed using Student's $t$-test and two-way repeated analysis of variance followed by a Dunnett's test. Values were considered significantly different at $P<0.05$.

HR before and after each ejaculation series in aged and young male rats is shown in Fig. 1. Only three of the aged rats ejaculated during copulatory behavior. There were significant differences in HR at rest (before exposure to the receptive female rat) between aged male rats $(322.4 \pm 13.6 \mathrm{bpm})$ and young male rats $(371.2 \pm$ $12.5 \mathrm{bpm})$ rats $(P<0.05)$. HR at rest was used a baseline value (set at $0 \%$ ). Increases above baseline in $\mathrm{HR}$ at 3 min before ejaculation, after introduction of the female rat, were $42.6 \pm 4.5$ and $20.2 \pm 3.5 \%$ for aged and young male rats, respectively. These values are significantly higher than the resting HRs $(P<0.01)$. In each age group, HR reached its peak at ejaculation (increases of $54.2 \pm$ 3.5 and $41.7 \pm 2.7 \%$, respectively), a level of which was significantly higher than the resting level $(P<0.01)$. HR at 1,2 , and $3 \mathrm{~min}$ after ejaculation in aged male rats were $40.6 \pm 4.6,33.0 \pm 4.7$, and $32.1 \pm 4.3 \%$ higher than the resting value, respectively (all significantly higher than the resting value; $P<0.01)$. HR of young male rats were $10.9 \pm 3.3,7.9 \pm 3.1$, and $13.0 \pm 3.2 \%$ higher than the resting value at 1,2 , and $3 \mathrm{~min}$ after ejaculation, respectively. The increase in HR at 3 min after ejaculation was still significantly higher than the resting HR. HR of aged male rats was significantly higher than that of young male rats at each time point $(P<0.05, P<0.01)$.

Decreases in HR after ejaculation of young and aged male rats are shown in Fig. 2. HR at ejaculation of aged male rats $(495.9 \pm 3.1 \mathrm{bpm})$ was significantly lower than that of young male rats $(524.3 \pm 9.4 \mathrm{bpm})(P<0.05)$. HR at ejaculation was used as an ejaculation value (set at $0 \%$ ). 


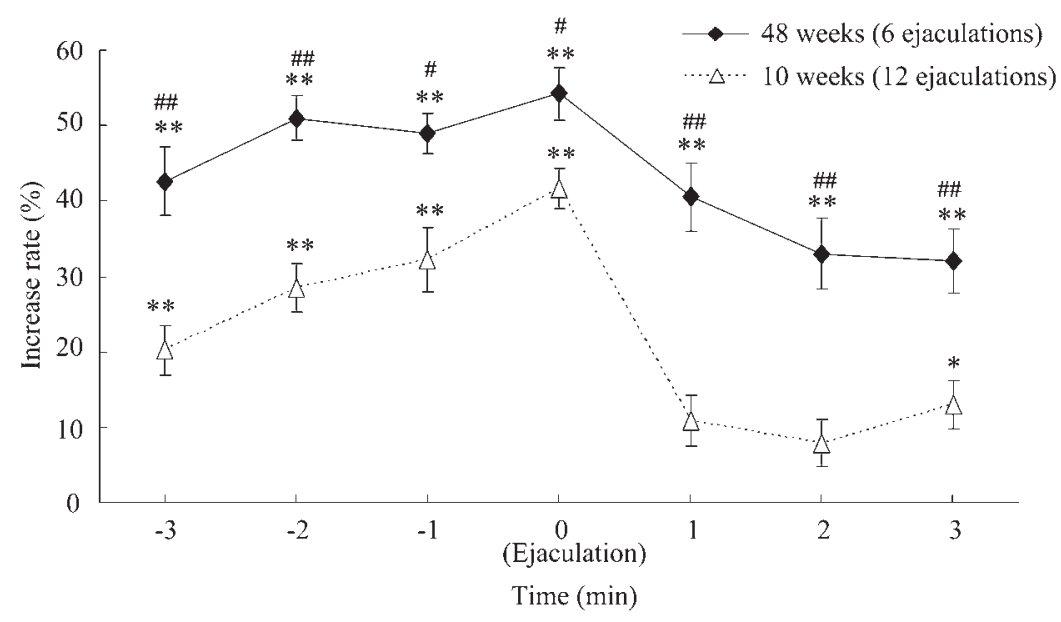

Fig. 1. Comparison of heart rate increases (HR) before and after ejaculations of male rats Change of HR during the $6 \mathrm{~min}$ centered on ejaculation (deemed $0 \mathrm{~min}$ ). $\mathrm{HR}$ at rest is the baseline value $(0 \%)$. All data represent mean \pm SE. Dunnett' test; *: $P<0.05$ vs rest time, **: $P<0.01$. $t$-test; ${ }^{\#}: P<0.05$ vs 10 weeks, \#\#: $P<0.01$ vs 10 weeks.

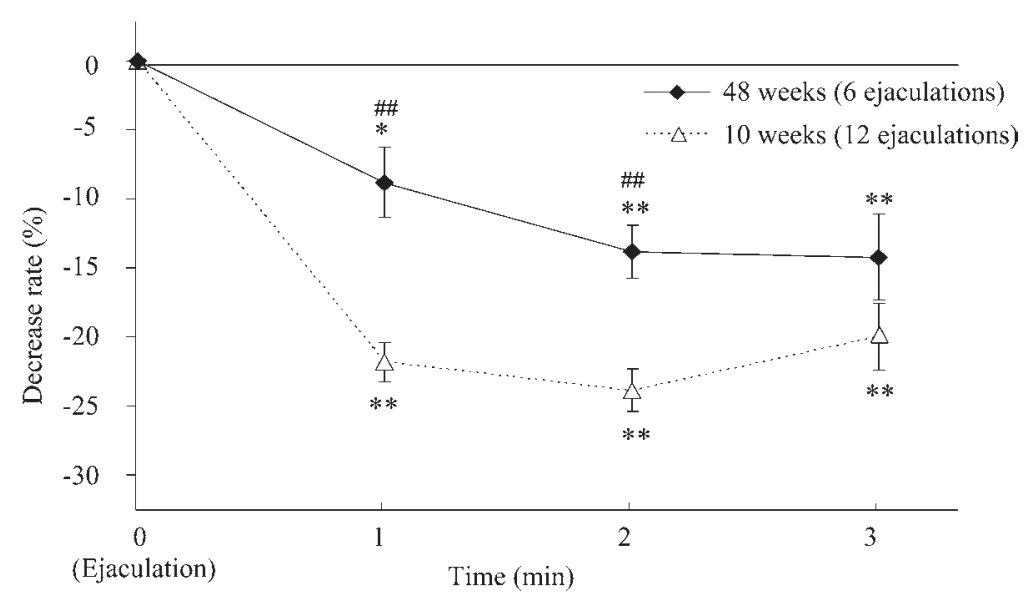

Fig. 2. Comparison of heart rate decreases (HR) after ejaculation of male rats. Change of HR for the $3 \mathrm{~min}$ after ejaculation. HR at ejaculation is given the value of $0 \%$. All data represent mean \pm SE. Dunnett' test; *: $P<0.05$ vs rest time, ${ }^{* *}: P<0.01$. $t$-test; ${ }^{\#}: P<0.05$ vs 10 weeks, ${ }^{\# \#}: P<0.01$ vs 10 weeks.

Decreases in HR from the ejaculation value at 1,2 , and 3 min after ejaculation of aged male rats were $-8.8 \pm 2.5$, $-13.8 \pm 1.9$, and $-14.2 \pm 3.1 \%$, respectively. These values were significantly lower than the ejaculation value $(P<0.05$ or $P<0.01)$. In young male rats, the corresponding HR values were $-21.8 \pm 1.4,-23.9 \pm 1.6$, and -20.0 $\pm 2.4 \%$, respectively, which were also significantly lower than the ejaculation value $(P<0.01)$. Following ejaculation, HR in aged male rats decreased more gradually than in young males, although the decreases in HR at 1 and 2 min after ejaculation of aged male rats was significant compared to those of young male rats $(P<0.01)$.

In the present study, HR during copulatory behavior reached its peak at ejaculation (Fig. 1) in both young and aged rats. These results support the findings of our previous study [26]. However, we did observe differences between the two age groups. The increase in HR at each time point was significantly higher in older male rats than in younger ones (Fig. 1). These results indicate that the aged heart has an added circulatory load during 
copulatory behavior, especially at ejaculation. Moreover, in young male rats, HR rapidly decreased after ejaculation. In contrast, the decrease was gradual in aged males (Figs. 1 and 2). These data suggest that there is a high load on the cardiovascular system after ejaculation. In humans, HR and systolic blood pressure during sexual behavior are more than twice the resting values [20]. In addition, these changes in HR and blood pressure can induce cardiac sudden death or subarachnoid hemorrhage $[9,21,29]$. On the basis of the results of our study, the risk of developing these disorders increases with age.

On the other hand, copulatory behavior in humans and animals declines with age $[5,8,10,11,18,22]$. A young male rat ejaculates approximately three times in $30 \mathrm{~min}$ [12], whereas 44- and 67-week-old male rats ejaculate approximately 1.6 and 1.0 times, respectively, during a 120 min period $[10,11]$. Male rats need to prepare quickly in order to perform the next ejaculation series $[1,2,6,7]$, however, because HR decreases more slowly after ejaculation in older rats, these animals are slower to perform, and they ejaculate less, during copulatory behavior. In aged male rats the decrease of HR after ejaculation was slower, compared with young males, and this may be the result of morphological and functional changes of the sinoatrial node $[13,24]$ and the decline of autonomic regulation during tachycardia and bradycardia $[3,4]$ with age.

In conclusion, the results of our study will assist in the improvement of reproductive technologies and the treatment and prevention of cardiac sudden death during male sexual behavior.

\section{Acknowledgment(s)}

The authors thank Professor Dr. L. Koranyi, Department of Clinical and Experimental Medicine, Semmelweis University, Budapest, Hungary, for his helpful advice. This study was supported in part by grants-in-aid for Scientific Research from the Society for Encouragement of Biomedical Education, a non-profit organization, to PK.

\section{References}

1. Anisko, J.J., Suer, S.F., McClintock, M.K., and Adler, N.T. 1978. J. Comp. Physiol. Psychol. 92: 821-829.

2. Barfield, R.J. and Geyer, L.A. 1975. J. Comp. Physiol.
Psychol. 88: 723-734.

3. Barringer, D.L. and Bunag, R.D. 1991. Mech. Aging Dev. 58: 111-125.

4. Barringer, D.L. and Bunag, R.D. 1991. Exp. Geront. 26 $65-75$.

5. Bishop, M.W. 1970. J. Reprod. Fertil. Suppl. 12: 65-87.

6. Blumberg, M.S., Mennella, J.A., and Moltz, H. 1987. Physiol. Behav. 39: 367-370.

7. Blumberg, M.S. and Moltz, H. 1987. Physiol. Behav. 40: 637-640.

8. Frankel, A.I. 1981. Horm. Behav. 15: 312-320.

9. Hellerstein, H.K. and Friedman, E.H. 1970. Arch. Intern. Med. 125: 987-999.

10. Hokao, R., Ito, T., Noguchi, J., Wakafuji, Y., Agematsu, Y., Saito, T.R., Takahashi, K.W., and Imamichi, T. 1992. Jikken Dobutsu 41: 259-268 (in Japanese).

11. Hokao, R., Saito, T.R., Wakafuji, Y., Takahashi, K.W., and Imamichi, T. 1993. Jikken Dobutsu 42: 75-82 (in Japanese).

12. Hokao, R., Saito, T.R., and Takahashi, K.W. 1993. Jikken Dobutsu 42: 451-455 (in Japanese).

13. Huang, X., Yang, P., Du, Y., Zhang, J., and Ma, A. 2007. Basic Res. Cardiol. 102: 429-435.

14. Ishii, K., Kuwahara, M., Tsubone, H., and Sugano, S. 1996. Lab. Anim. 30: 7-12.

15. Kinsey, A.C., Pomeroy, W.B., and Martin, C.E. 1948. Sexual Behavior in The Human Male, W.B. Saunders, Philadelphia.

16. Krüger, T., Exton, M.S., Pawlak, C., von zur Mühlen, A., Hartmann, U., and Schedlowski, M. 1988. Psychoneuroendocrinology 23: 401-411.

17. Krüger, T.H., Haake, P., Chereath, D., Knapp, W., Janssen, O.E., Exton, M.S., Schedlowski, M., and Hartmann, U. 2003. J. Endocrinol. 177: 57-64.

18. Larsson, K. 1958. J. Gerontol. 13: 136-139.

19. Lee, S., Chae, J., and Cho, Y. 2006. J. Korean Med. Sci. 21: 995-999.

20. Littler, W.A., Honour, A.J., and Sleight, P. 1974. J. Reprod. Fertil. 40: 321-331.

21. Locksley, H.B. 1966. J. Neurosurg. 25: 219-239.

22. Minnick, R.S., Warden, C.J., and Arieti, S. 1946. Science 103: 749-750.

23. Nalbantgil, I., Yigitbasi, O., and Kiliccioglu, B. 1976. Am. Heart J. 91: 405-406.

24. Regina de Melo, S., Rodrigues de Souza, R., and Mandarimde-Lacerda, C.A. 2002. Biogerontology 3: 383-390.

25. Saito, T.R., Arkin, A., and Takahashi, K.W. 1999. Scand.J. Lab. Anim. Sci. 26: 79-82.

26. Saito, T.R., Hokao, R., Terada, M., Takahashi, K.W., Tsubone, H., and Sugano, S. 2001. Scand. J. Lab. Anim. Sci. 28: 108-113.

27. Saito, T.R., Terada, M., Moritani, N., Hashimoto, H., Oikawa, T., and Soeta, S. 2003. Exp. Anim. 52: 153-157.

28. Terada, M., Momozawa, Y., Komano, M., Kusunose, R., Sato, F., and Saito, T.R. 2005. Reprod. Med. Biol. 4: 143-147.

29. Ueno, M. 1963. Nihon Hoigaku Zasshi 17: 330-340 (in Japanese). 